\title{
On linear internal waves on the sea, strongly vertically trapped
}

\author{
R. Putrini $(*)-$ E. Salusti $(* *)$
}

Received on August 2nd, 1975

\begin{abstract}
Sumart. - We study some explicit cases of marine thermocline. We focus our attention on the strongly vertically trapped internal waves, which in our cases allow an explicit dispersion relation and a simple behaviour in terms of elementary functions. The explicit form of the Vaisala-Brunt frequency $N^{2}(z)$ is proportional to $1 /\left|z-z_{0}\right|$ in one case and to $A^{2}-B^{2}\left(z-z_{0}\right)^{2}$ in the other. A comparison with some experimental data concerning the Ijigurian Sea is actually in course.

Riassuxto. - In relazione a determinate condizioni di superficie, la struttura verticale del mare si caratterizza meliante una brusca variazione nella densita. Nel presente lavoro vengono studiate le onde interne che vi risultano fortemente intrappolate, ottenendo relazione di dispersione, velocita di gruppo e correlazione in termini di funzioni elementari per the situazioni sperimentali individuabili analiticamente attraverso la frequenza di VäisäläBrunt $N^{2}(z)$ proporzionale a $1 /\left|z-z_{0}\right|$ in un caso ed uguale a $A^{2}-B^{2}\left(z-z_{0}\right)^{2}$ nell'altro. $\hat{E}$ in corso un confronto con i dati provenienti da campagne di misura effettuate nel Mar Ligure.
\end{abstract}

\section{1. - I.NTRoduction}

An interesting problem in the energy balance of a sea, concerns the internal waves and their energies. These are waves which propagate horizontally and their largest amplitude is related to the vertical variations of the density $o(z)$. This is due to the vertical variations of temperature and salinity, which are originated by the intense ex-

${ }^{*}$ ) C.N.R. - Istituto Fisica dell Atmosfera - Roma, Italy.

(**) Istituto di Fisica "G. Marconi", Universita di Roma, Italy. Istituto di Fisica Nucleare - Sezione di Roma 
change of moment and heat with the mowing atmosphere. More in detail, one could first separate a "mixed layer" which has a vertical extension of some tens of meters uncler the air-sea surface. This layer is mixer by the turbulence propagating downward from the moving atmosphere (this is one of the eflects of winds, storms, atmospherie turbulence etc.). Its energy propagates downward freely and makes temperature, salinity, density and motion-homogeneous in this "mixerl layer". The surface under this mixed layer is the natural clomain of propagation for the most intense internal waves. It has however to be remarked that it is not very easy to distinguish what percentage of atmospherie energy generates internal waves and what percentage of energy is used by the system to erode the underlying stratifierl region. Practically, moreover, one can remark that many times the mixerl layer has not a very sharp division with the underlying stratified region, but lass a vertical extension of $50 \div-500$ meters. The resulting periodic phenomena, the internal waves, are in this case related to a smoother variation of the density than in the case of the sharp division between the mixed layer and the deeper stratified region.

Tts stratification is a rather curious phenomenon: one can experimentally remark many sheets of an horizontal extension of kilometers and this is a surprising contrast with the vertioal extension of few centimeters. Practically, forgetting this "fine-structure", one could see it as a stable region of slowly varying rensity $\varrho_{\mathrm{o}}(z)$.

In the lowest part, $\varrho_{0}(z)$ decreases with the depth as a slow exponential exp $(-\alpha z)$, where $\alpha$ is a constant.

In this contest, we have remarkerl that the surface between the mixerl layer and the stratified thermocline is the domain of many interesting and important phenomena, related to the internal waves distribution of energy insirle the fluid (i). It can be shown, more prerisely, that the knowledge of exact shape of $g_{0}(z)$, the static density profile, could give essential informations concerning the internal waves structure, their correlations and their energetics.

More explicitly, it has to be adrler that the experimental evidence stresses that this is not really a sharp surface, but it appears rather as a vertical region of transition between the homogeneous mixerl layer and the stratified thermocline. This appears interesting because the internal waves can be described by a simple equation (if one assumes the linear waves and if the Boussinesq approximation is assumed valial: see, for example, Phillips $\left(^{(}\right)$and Thorpe $\left({ }^{7}\right)$ ) where the explicit shape of $Q_{0}(z)$ plays an explicit role. 
Now, this equation has simple dispersion relation and solution in some cases rather well known in the literature. These are supplied when the Vaisala-Brunt frequency

$$
N^{2}(z)=-\frac{\mathrm{d} \varrho_{0}}{\mathrm{~d} z} \cdot \frac{g}{\varrho_{\mathrm{o}}}>0
$$

has a $\delta(z)$ behaviour or $N-(z)=$ const. behaviour (Phillips $\left({ }^{6}\right)$ ) and when

$$
N^{2}(z)= \begin{cases}0 \quad \text { for } 0<z<d & (d \text { is the depth of the discontinuity } \\ e^{-\alpha z} \text { for } z>d & \text { in the density })\end{cases}
$$

as in the classical analysis of Garret and Munk $\left(^{3}\right)$ and in few other cases studied by Thorpe $\left({ }^{8}\right)$.

The realistic cases are rather different; one could easily solve them numerically, but this would imply some loss of informations. For this region we have studied two rather realistic profiles

$$
N^{2}(z)=\alpha^{2} /\left|z-z_{0}\right| \text { and } N^{2}(z)-A^{2}-B^{2}\left(z-z_{0}\right)^{2}
$$

These profiles can, in some cases, simulate correctly the experimental situation and they also allow an explicit calculation of the internal waves, their dispersion r'elation, their group and wave velocities, their correlations.

An experimental verification is actually in course $\left(^{1}\right)$.

\section{LINEAR THEORY OF MARINE INTERNAL WAVES}

The theory of internal waves is rather well known (Phillips $\left(^{6}\right)$, Thorpe $\left({ }^{8}\right)$ ). In the following we will repeat the essential results on the time evolution of these waves.

In fact, in the case that the Boussinesq approximation can be assumed and the earth's rotation can be disregarded, the velocity components satisfy the equations $(6,7)$.

$$
\begin{gathered}
\partial_{t} u+\frac{1}{\varrho_{0}} \frac{\partial p}{\partial w}=0 \\
\partial_{t} v+\frac{1}{\varrho_{0}} \frac{\partial p}{\partial y}=0 \\
\partial_{t} w+\frac{1}{\varrho_{0}} \frac{\partial p}{\partial z}+g \frac{\varrho^{\prime}}{\varrho_{0}}=0
\end{gathered}
$$


where $p$ is the departure from the hydrostatic pressure and one has assumed

$$
\varrho=\varrho_{0}(z)+\varrho^{\prime}(x, y, z, t)
$$

with the density variation $\varrho^{\prime} \ll \varrho_{0}$.

In the same frame of approximation, one also assumes that the water is incomprensible:

$$
\begin{gathered}
\partial_{x} u+\partial_{y} v+\partial_{z} v=0 \\
\frac{d \varrho}{r i t}-\frac{\partial \varrho^{\prime}}{\partial t}+\pi \frac{\partial \varrho^{\prime}}{\partial x}+\because \frac{\partial \varrho^{\prime}}{\partial y}+w \frac{\partial \varrho_{o}}{\partial z}=0
\end{gathered}
$$

Calculating first the time derivative of the vorticity, one has

$$
\begin{aligned}
& \partial^{2} t t\left(\frac{\partial u}{\partial z}-\frac{\partial w}{\partial x}\right)+\frac{\partial}{\partial t} \frac{g}{\varrho_{0}} \frac{\partial \varrho^{\prime}}{\partial x}=0 \\
& \partial^{2}{ }_{\iota t}\left(\frac{\partial v}{\partial z}-\frac{\partial w}{\partial y}\right)+\frac{\partial}{\partial t} \frac{a}{\varrho_{0}} \frac{\partial o^{\prime}}{\partial y}=0
\end{aligned}
$$

and, taking into account the $\frac{\mathrm{d} o}{\mathrm{~d} t}=0$ relation, one has

$$
\begin{aligned}
& \frac{\partial}{\partial t} \frac{g}{\varrho_{0}} \frac{\partial \varrho^{\prime}}{\partial x}=\frac{g}{\varrho_{0}} \frac{\partial}{\partial x} \frac{\partial}{\partial t} \ddot{ }^{\prime}=-\frac{g}{\varrho_{0}} \frac{\mathrm{d} \varrho_{0}}{\mathrm{~d} z} \frac{\partial w}{\partial z} \\
& \frac{\partial}{\partial t} \frac{g}{\varrho_{0}} \frac{\partial \varrho^{\prime}}{\partial y}=\frac{g}{\varrho_{0}} \frac{\partial}{\partial y} \frac{\partial}{\partial t} \varrho^{\prime}=-\frac{g}{\varrho_{0}} \frac{\mathrm{d} \varrho_{0}}{\mathrm{~d} z} \frac{\partial w}{\partial y}
\end{aligned}
$$

Taking then the horizontal divergence, one has the basic relation $\partial^{2} u\left(\partial^{2} x x+\partial^{2} y y+\partial_{z z}\right) v(\mathrm{x}, z, t)+N^{2}(z)\left(\partial^{2}{ }_{x x}+\partial^{2}{ }_{y y}\right) w(\mathrm{x}, z, t)=0[2.1]$ where $N(z)=-\frac{g}{\varrho_{0}} \frac{d}{d} z \quad \epsilon_{c}>0$ is the Väisäla-Brunt frequency.

Assuming a plane progressive wave solution of the form

$$
w(\mathrm{x}, z, t)=W(z) \exp i\left(K_{x} x+K_{y} y+K_{z} z-\omega t\right)
$$

one easily arrives to the equation

$$
\frac{\mathrm{d}^{2} W(z)}{\mathrm{d} z^{2}}+\left(-\frac{N^{2}(z)}{w^{2}} K^{2}-K^{2}\right) W(z)=0
$$


with

$$
\begin{array}{ll}
W(0)=0 & \text { at the rigid free surface }\left({ }^{9}\right) \\
W(-d)=0 & \text { at the bottom }
\end{array}
$$

The informations concerning the stratification are included in the particular shape of $N^{2}(z)$. This is rather constant in the mixed layer. In the lower region it has many sharp variations (the fine-structure).

These variations give the classical behaviour of $N^{2}(z)$ in the thermocline when, in some sense, they are averaged in $z$. At last $N^{2} \longrightarrow$ const. value in the deepest regions can be found.

As the varions preceding cases have been studied, we have focused our attention to two explicitly solvable cases:

$$
\begin{array}{ll}
\text { a) } & N^{2}(z)=\alpha^{2} /\left|z-z_{0}\right| \\
\text { b) } & N^{2}(z)=A^{2}-B^{2}\left(z-z_{0}\right)^{2}
\end{array}
$$

where $\alpha, A, B$, are constants to be determined on experimental ground. The depth $z_{0}$ is that corresponding to the zone of highest variation of the Vaisala-Brunt frequency $N^{2}(z)$. The cases seem to be general enough to approximate realistic cases, particularly in the parabolic case.

\section{ThE EXPLICIT CASE $N^{2}(z)=\alpha^{2} /\left|z-z_{0}\right|$}

We are now going to study the case above mentioned $N^{2}(z)=a^{2} /\left|z-z_{0}\right|$. The equation [2.2] now results

$$
\frac{\mathrm{d}^{2} W(z)}{\mathrm{d} z^{2}}=\left(K^{2}-\frac{K^{2} \alpha^{2}}{\omega^{2}} \frac{1}{\left|z-z_{0}\right|}\right) W(z)
$$

We assume that the distance among $z_{0}$, the bottom $z=-d$ and the surface $z=0$ could be considered large. In practice $z_{0}$ is 20-50 meters for localized seas ( $\left.{ }^{1}\right)$ and hundred meters for the Ocean. The depth d of the bottom is usually fixed to be larger, in order to avoid bottom effects. For strongly trapped internal waves, the vertical region of interest is determined in order to fix the vertical scale of motion. It usually is of the order of magnitude of few meters. Outside this region $z=z_{0}$, the Vaisala-Brunt frequency decays rather rapidly, as a power of $z$. Much more quick, however, is the decay of the solution W, which results in general a negative exponential. So one could also assume for these waves an idealized boundary condition

$$
W( \pm \infty)=0
$$


which simplifies the calculations. Then one can say that our eigenvalue equation can assume an infinity of eigensolutions, labelled by $\mu=1,2,3 \ldots$

The general solution is (see Appendix):

$$
W_{\mu}=A_{\mu} \varrho^{-K\left(z-z_{0}\right)} L_{\mu-1}^{1}\left\{2 K\left|z-z_{0}\right|\right\}
$$

where $A_{\mu}$ is a constant and the function $L_{\mu_{-1}}^{1}(\varrho)$ is called Laguerre polynomial. At $z=z_{0}$, the function is complicated: the equation shows that its second derivative diverges. Then $W_{\mu}$ results a continuous function symmetric around the peak $z_{0}$ One can moreover say (see Appendix) that the solution exist if and only if

$$
\frac{\alpha^{2} K}{2 \omega^{2}}=\mu=1,2, \ldots
$$

It's interesting to note that the preceding dispersion relation for these internal waves is similar to that of the two-fluid system.

Then one can calculate the group velocity $c_{g}$

$$
c_{g}=\frac{d \omega}{d K}=\frac{1}{2} \cdot \frac{a^{2}}{\mu(2 h)^{1 / 2}}
$$

One has also to remark that $W_{\mu}$ is proportional to $\exp -K\left|z-z_{0}\right|$ so that the behaviour of the wave decays exponentially outside the zone of sharp variation of $N^{2}(z)$.

This implies that we must consider only waves with a large $K$ value fixed by the dimension of the physically interesting region through the relation $K \sim 1 / L$. One could enlarge the preceding treatment of the individual structure of internal waves by considering the correlation function. This quantity is

$$
B_{\mu \mu^{\prime}}(K)=\overline{W_{\mu} W_{\mu}^{\prime}}=\int_{-\infty}^{+\infty} W_{\mu} W_{\mu \mu^{*}}^{*} \mathrm{~d} z^{\prime}
$$

It appears interesting because it is a powerful tool in the comparison between theoretical models and experimental data $\left({ }^{4}\right)$. In our case it results:

$$
B_{\mu \mu^{\prime}}(K)=-\frac{1}{2 K L} \int_{0}^{L / 2} A_{\mu} A_{\mu \mu^{\prime}}^{*} e^{-2 K\left(z-z_{0}\right)} L_{\mu-1}^{1} L_{\mu^{\prime}-1}^{1 *} d\left\{2 K\left|z^{\prime}-z_{0}\right|\right\}
$$


The Laguerre polynomial are on orthogonal set, therefore one has

$$
B_{\mu \mu^{\prime}}(K)= \begin{cases}0 & \text { for } \mu \neq \mu^{\prime} \\ \frac{1}{2 L}\left\{\left|A_{\mu}\right|(\mu-1) !\right\}^{2} K^{-1} & \text { for } \mu=\mu^{\prime}\end{cases}
$$

this $K^{-1}$ behaviour of the correlation function is rather interesting and is in agreement with the results of Phillips $\left(^{6}\right)$ for a range of the spectrum of the lowest internal mode. If one wants to calculate also the cross-correlation $\overline{W V}$, the continuity equation

$$
i K_{y} V+\frac{\lambda}{i z} W=0
$$

must be used. This can give the matrix element

$$
\int\left(\frac{\mathrm{d} W}{\mathrm{~d} z}\right) W \mathrm{~d} z^{\prime}=0
$$

One could add that the matrix element

$$
\int\left(\frac{\mathrm{d} W_{n}}{\mathrm{~d} z}\right) W_{m} \mathrm{~d} z^{\prime}
$$

is not zero if (and only if) $n=m \pm 1$. In more detail

$$
\int\left(\frac{\mathrm{d} W_{n}}{\mathrm{~d} z}\right) W_{n-1} \mathrm{~d} z^{\prime}=1 / \frac{n}{2}
$$

and

$$
\int\left(\frac{\mathrm{d} W n}{\mathrm{~d} z}\right), W_{n+\mathrm{i}} \mathrm{d} \vartheta^{\prime}--\frac{\sqrt{n+1}}{2}
$$

To finish, also the case

$$
N^{2}(z)=\frac{\alpha^{2}}{z}-\frac{\beta z}{z^{2}} \quad \alpha, \beta \text { const. }
$$

could be exactly treated. It results, however, of different physical interest because it describes an instable case, whereas in this note one studies stable phenomena only. 
4. TIIE CASE OF a PARABOLA $N^{2}(z)=A^{2}-B^{2}\left(z-z_{0}\right)^{2}$

By repeating the preceding considerations, one can arrive to the equation:

$$
\frac{\mathrm{d}^{2} W(z)}{\mathrm{d} z^{2}}=\frac{R^{2} K^{2}}{\omega^{2}}\left(z-z_{0}\right)^{2} W(z)-\left\{\frac{A^{2} K^{2}}{\omega^{2}}-K^{2}\right\} W(z) ; A^{2}>\omega^{2}
$$

with the boundary conditions (6),

$$
\begin{aligned}
& W(0)=0 \\
& W(-d)=0
\end{aligned}
$$

In this case also there is an infinity of solutions (see Appendix):

$$
W_{q}=A_{q} e^{-\frac{B K}{2},\left(z \cdot z_{0}\right)^{-}} H_{q}\left\{\left(\frac{K B}{\omega}\right)^{1 / 2}\left(z-z_{0}\right)\right\} \quad q=1,2, \ldots
$$

Where $A_{q}$ is a constant and $H_{q}(o)$ is an Hermite polynomial. From this equation one can see that the wave amplitude decreases more rapidly than in the preceding case (eq. [3.2]) as $\left|z-z_{0}\right|$ increases.

There is also in this case a dispersion relation

$$
2 q+1=\frac{A^{2} K}{\omega B}-\frac{K(1)}{B}
$$

It has to be remarked that a decreases as $q$ increases, that is, for higher morles, the dimension $L$ of the system fixes a $K$ value $K \sim 1 / L$. From the above equation, one can derive:

$$
(i)=\frac{-C B+\left(C^{2} B^{2}+4 K^{2} A^{2}\right)^{1 / 2}}{2 K} \quad C=q+1
$$

and then the group velocity can be deduced

$$
c_{g}=\left\{\frac{2 A^{2}}{\left(C^{2} B^{2}+4 A^{2} K^{2}\right)^{1 / 2}}+\frac{1}{2 K^{2}}\left[C B-\left(C^{2} B^{2}+4 A^{2} K^{2}\right)^{1 / 2}\right]\right\}
$$

In this case also it is easy to calculate the correlation function

$$
B_{q q^{\prime}}(K)=\frac{1}{L} \int_{0}^{L / 2} A_{q} A_{\sigma^{\prime}}^{*} e^{-\frac{K B}{\omega}\left(z^{\prime}-z_{0}\right)^{2}} H_{q} H_{q^{\prime}}^{*} \mathrm{~d} z^{\prime}=
$$




$$
\left.=\frac{1}{L}\left(\frac{\omega}{K B}\right)^{1 / 2} \int_{0}^{L / 2} A_{q} A_{q^{\prime}}^{\mathrm{*}} H_{q} H_{q^{\prime}}^{\prime} e^{-\frac{K B}{\omega}\left(z^{\prime}-z_{0}\right)^{2}} \mathrm{~d} \mid\left(\frac{K B}{\omega}\right)^{1 / 2}\left(z^{\prime}-z_{0}\right)\right]
$$

which, remembering the orthogonality of the Hermite polynomials, becomes

$$
B_{q q^{\prime}}(K)= \begin{cases}0 & \text { for } q \neq q^{\prime} \\ \left|A_{q}\right|^{2}\left(\frac{\pi \omega}{K B}\right)^{1 / 2} \frac{2^{q}}{L} q ! & \text { for } q=q^{\prime}\end{cases}
$$

This result can be written in a more clear form by using the dispersion relation.

In fact, from eq. [4.2] one has that

$$
\omega K^{-1} \sim K^{-2}
$$

for which eq. [4.3] becomes

$$
B_{q q^{\prime}}(K) \propto K^{-1 / 2}
$$

In this case, the behaviour of the correlation function is different from the other one.

$\Lambda s$ in the precerling section, if one wants to calculate the correlation $\overline{W V}$, one must use the continuity equation, giving $V$ as a function of $\frac{\mathrm{d} W(z)}{\mathrm{d} z}$. Now, the resulting matrix element

$$
\int\left(\frac{\mathrm{d} W\left(z^{\prime}\right)}{\mathrm{d} z^{\prime}}\right) W \mathrm{~d} z^{\prime}
$$

ean be found in the literature $\left(^{5}\right)$.

The profile

$$
N^{2}(z)=A^{2}-B^{2}\left(z-z_{0}\right)^{2}+C\left(z-z_{0}\right)+\mathrm{D}\left(z-z_{0}\right)^{3}
$$

can also be calculated. The result is exact for $C \neq 0$ and with some approximation for $D \neq 0$.

To finish, we want to note that results obtainerl in this note for the internal waves in the presence of a strong stratification are user for a comparison with experimental clata concerning the Ligurian sea (1). 


\section{APPENDIX}

a) $T h e \frac{\alpha^{2}}{\left|z-z_{0}\right|}$ case.

This equation is rather well known. We follow here a description of the solution which is widely used in quantum mechanies $\left({ }^{2}\right)$. In adimensional form, it results

$$
\frac{\mathrm{d}^{2} u}{\mathrm{~d} \varrho^{2}}+\left(\frac{\lambda}{\varrho}-\frac{1}{4}\right) u=0
$$

Asimptotically the solution results

$$
u \sim e \pm \varrho / 2
$$

and if one wants $u$ to be finite,

$$
u \sim e^{-\varrho / 2}
$$

Multiplication by a polynomial doesn't change the asimptotic value

$$
u=F(\varrho) e^{-\varrho / 2}
$$

This gives

$$
\frac{\mathrm{d}^{2} F}{\mathrm{~d} \varrho^{-}}-\frac{\mathrm{d} F}{\mathrm{~d} \varrho}+\frac{\lambda}{\varrho} F=0
$$

Putting now

$$
F=\sum_{K=1} A_{K} Q^{K}
$$

inside the precerling equation, one has

$$
\begin{gathered}
(\lambda-1) A_{1}+2 A_{2}=0 \\
\cdot \cdot \cdot \cdot \\
\cdot(K+1) A_{K+1}+(\lambda-K) A_{K}=0
\end{gathered}
$$

Now, if one assumes that the series is infinite, then

$$
\frac{A_{K+1}}{A_{K}}=\frac{K-\lambda}{K(K+1)} \underset{K \rightarrow \infty}{\longrightarrow} \frac{1}{K}
$$

which correspond to an exponential behaviour of $F(\varrho)$, is in contradiction with our hypothesis $u \sim e^{-0 / 2}$. 
Then the series cannot be infinite.

The other only possibility is that $\lambda$ be an integer $n$.

Then one has the "eigenvalue condition"

$$
\begin{aligned}
& \lambda=n \\
& A_{K}=0 \quad K>n
\end{aligned}
$$

The polynomials obtained in this way are related to the associated Laguerre polynomials:

$$
L_{n}^{1}(\varrho)=e^{\varrho} \frac{\mathrm{d}^{n}}{\mathrm{~d}^{n}}\left(\varrho^{n} e^{-\varrho}\right)
$$

The first one are:

$$
\begin{gathered}
L^{1_{0}}=1 ; \quad L_{1}=4-2 \varrho ; \quad L^{1}{ }_{2}=18-18 \varrho-3 \varrho^{2} \\
L^{1_{3}}=96-144 \varrho+48 \varrho^{2}-4 \varrho^{3}
\end{gathered}
$$

b) The $A^{2}-B^{2}\left(z-z_{0}\right)^{2}$ case

We repeat here the precerling treatment of the equation. In arlimensional form

$$
\frac{\mathrm{d}^{2} \cdot t}{d^{2}}+\left(\lambda-g^{2}\right) u=0
$$

The asimptotic value being

$$
u \sim e^{-\varrho^{2} / 2}
$$

If one assumes

$$
u=H(\varrho) e^{-\varrho^{2} / 2}
$$

then obtains, for the basic equation

$$
\frac{d^{2} H}{d \varrho^{2}}-2 \varrho \frac{d H}{d \varrho}+(\lambda-1) H=0 .
$$

Now, writing for $H$

$$
H=\sum_{n=1}^{N} a_{n} \varrho^{n}
$$

the dilferential equation [i.1] gives

$$
\frac{a_{s+2}}{a_{s}}=\frac{(2 s+1)-\lambda}{(s+2)(s+1)} \quad s \geqslant 0
$$


Separating the even or add solutions, on then has

$$
\lambda=2 n+1
$$

The solutions are even or ard polynomials, the Hermite polynomials

$$
H_{q}(\varrho)=(-1)^{q} e^{\varrho / 2} \frac{\mathrm{d}^{n} \rho^{-} \varrho^{2}}{\mathrm{~d} \varrho^{n}}
$$

The first one are

$$
H_{0}=1 ; \quad H_{1}=2 \varrho ; \quad H_{2}=4 \varrho^{2}-2 ; \quad H_{3}=8 \varrho^{3}-12 \varrho
$$

\section{REFERENCES}

(1) Colacino Ml., Purisi R., Roveldi A., Stoccinno C., 1976. - Sludio del bilancio termico diurno del ciclo di temperatura e delle onde interne in stazione fissa (Mar Ligure). Nota interna dell'Istituto Idrografico della Marina, Genova.

(2) Dicke R., Witrke J., 1960. - Introduction to Quantum Mechanics. "Addison Wesley, Publ. Comp. Inc.", London.

(") Garket C., Munk W., 1972.-Space-Time Scale of Internal Waces. "Geopl. Fluid Dyn.", 2, pp. 225-264.

(4) Monix A. S., Yagion A. I., 1971. - Statistical Fluid Mechmics. "The Mit Press", I.

(5) Monse P', Fesnibach H., 1953. - Methods of Theoretical Physics. "MeGraw Hill".

(6) P'HILlips O. M., 1966. - The Iynamie of the Upper Ocean. "Cambrirlyo University Press".

(7) SEe the classical discussion in the book of Phillips, Reference $\left({ }^{6}\right)$.

$\left.{ }^{8}\right)$ Thonpe S. A.; On the Shape of Progressive Internal Waves. "Royal Soc., London, Philos. Trans"., V 263 A; II 45; pp. 563-614.

(") THompe S. A. (and other arlicles), 1975. - "J. of Geoph. Research", 80. 